\title{
Transient Analysis and Safety Assessment of Turbofan Engine Structures during Bird Ingestion
}

\author{
Gang Luo, ${ }^{1,2}$ Chi Ma, ${ }^{1,2}$ Wei Chen $\mathbb{D}^{1,2}$ Lulu Liu, ${ }^{1,2}$ and Zhenhua Zhao ${ }^{1,2}$ \\ ${ }^{1}$ College of Energy and Power Engineering, Nanjing University of Aeronautics and Astronautics, Nanjing 210016, China \\ ${ }^{2}$ Aero-Engine Thermal Environment and Structure Key Laboratory of Ministry of Industry and Information Technology, \\ Nanjing 210016, China
}

Correspondence should be addressed to Wei Chen; chenwei@nuaa.edu.cn

Received 25 December 2019; Revised 28 May 2020; Accepted 1 July 2020; Published 17 July 2020

Academic Editor: Zhiguang Song

Copyright (c) 2020 Gang Luo et al. This is an open access article distributed under the Creative Commons Attribution License, which permits unrestricted use, distribution, and reproduction in any medium, provided the original work is properly cited.

\begin{abstract}
The high bypass ratio turbofan engine's load-carrying structure transient response during bird ingestion was analyzed in accordance with the engine bird ingestion certification regulations, the principles of structural safety assessment were represented, and the structural safety analysis and assessment method of Turbo-Fan engine during bird ingestion were proposed. A high bypass ratio turbofan engine's FEM was established and verified the rationality when its' operation. Large bird ingestion into an engine's procedure was conducted, the dynamic responses of key components on engine's load-carrying structures during the bird ingestion were discussed, and the safety assessment consequence was obtained. We draw a conclusion that the relevant analysis/simulation data could be submitted to engine certification administration as key documents, the structural safety analysis and assessment method of turbofan engine due to bird ingestion could be applied as analysis and prediction work in the engine bird ingestion certification.
\end{abstract}

\section{Introduction}

High bypass ratio turbofan engine often encounters bird ingestion, which affects the structural safety of the engine [1-3]. After the birds are being ingested into the engine, the bird's carcasses may impact the fan/compressor blade, which not only causes the bending, bulging, and tearing of the leading edge of the blade but also leads to more serious failure of the whole engine. The bird ingestion accidents of JT9D engine in 1997 [4] and PW4060 engine in 2007 [5] show that during bird ingestion of high bypass ratio turbofan engine, a sudden increase of high-energy load will occur in the structure, which would cause complex dynamic response in the key components of its transmission path, thus endangering the structural safety of the whole engine. Aiming at the structural safety of the whole engine when ingesting birds, airworthiness regulations and supporting advisory circulars are adopted to regulate the minimum structural safety requirements of the engine bird ingestion, as well as the guidelines for the analysis, simulation, and test studies that must be carried out to adapt the structural safety requirements [6-8], and related studies are carried out mainly for the impact damage. At present, the research on the structural response of engine on bird ingestion mainly focuses on the following two aspects: the bird impact damage of fan blade and the structural dynamics of engine after bird ingestion.

Castelletti and Anghileri [9] considered the effect of cutting force; the numerical calculation of different sizes of artificial bird was carried out and compared with the impact test. The analysis showed that both cylinder and ellipsoid artificial bird are suitable for the calculation and analysis of bird impact blade. Frischbier and Kraus [10] simulated a 2.5pound-bird ingestion into a pw6000 turbofan engine by ALE and SPH methods; the loads and mechanical responses of the front-end high-pressure compressor were predicted; Yupi et al. [11] carried out a bird impact test of real fan blades and measured the dynamic response of each characteristic position of the blade during bird impact. Mao et al. [12-14] and Shmotin et al. [15] used different constitutive models and state equations to study the dynamic response and damage of bird impact blade. Selezneva et al. [16] simulated the bird impact fan blade by using the artificial bird with an 
aspect ratio of 1.5; the results were in good agreement with the experimental data.

Prakash [17] showed that the fan blades with shoulders had better impact resistance and improved the energy absorption of the fan rotor significantly. Vignjevic et al. [18] carried out the parametric studies on bird impact fan blade, which includes influence of the bird shape, the bird impact location, and impact time. Simulation results from these studies were compared and validated against the final deformed shape of the blade recovered from the bird impact test. Samuel et al. [19] made simulations of jet engine bird ingestion and comparison of rotating to nonrotating fan blade dynamic response due to bird impact. Siddens and Bayandor [20] took a research about engine hybrid structural damage due to bird impact, including fan blade and casing, and developed a multidisciplinary technique to form a full multiphysics, multiscale crashworthiness analysis methodology in accordance with the bird ingestion. In another publication, Siddens and Bayandor [21] established a finite element model including the complete stage fan blade, casing, and rotating shaft, studied the damage of the fan blade after bird impact in the running state, and carried out a suitable prediction of the initial damage of the blade and component failure during the engine bird impact design. Liu and $\mathrm{Li}$ [22] built a rotary engine primary compressor bird impact model and studied bird impact whole stage blades' damage and dynamic responses by using a validated bird model. Handschuh et al. [23] used gelatin bird to impact the leading edge of a composite blade. It was found that the staggered toughening method could enhance the resistance to damage of the leading edge. Sandi et al. [24] presented a bird strike test article design, subcomponent blade leading edge fabrication, test method development, and initial results from ballistic impact of a gelatin projectile on the leading edge of composite fan blades. The simplified test article geometry was developed to realistically simulate a blade leading edge while decreasing fabrication complexity. Impact data was presented on baseline composite blades and toughened blades. Zeng et al. [25] research described the TC4 hollow fan blade structural optimization based on bird strike analysis; bird strike on a plate was simulated first to validate the algorithm, and the effect of parameters on results in bird material model was discussed; then, bird strike on a solid blade and a hollow blade was simulated to see how hollow structure affects crashworthiness; finally, geometric optimization for hollow fan blade based on crashworthiness was conducted with the method of orthogonal experiment. Zhang and Fei [26] presented the effect of bird geometry and impact orientation in bird striking on a rotary jet engine fan analysis using the SPH method; the research work indicated that bird geometry and impact orientation had a significant effect on the impact force, impact duration, and kinetic energy loss of the bird. As a comprehensive analysis of specific numerical simulation methods, Sinha [27] summarized a variety of numerical simulation methods used in the analysis of birdslicing impact, including Lagrange, ALE, and SPH methods, and found that the SPH method can better characterize the physical and mechanical processes of fan blade bird ingestion.
Literatures on structure safety analysis and assessment of bird ingestion are less reported. Ramachandra's study [28] showed that bird impact at the tip of the blade during takeoff might causes an axial load with a peak value of about $20 \mathrm{kN}$ on the front fulcrum bearing of the fan. Czeslaw [29] established a finite element model of the whole structures for the midbird impact engine and carried out the simulation of the impact process of high-energy loads such as bird impact and blade off. Liebich [30] designed and developed a test rig that represented the rotor dynamic behavior of an aeroengine almost in a full size scale, which could study the unbalance response of the whole engine model. Badshah et al. [31] established the rotor parts including the fan, compressor, and turbine, carried out the analysis of bird impact fan based on the SPH method, and obtained the impact response of the fan. Then, the modal analysis of the rotor parts with bird impact deformation was carried out at different rotational speeds, and it was found that the change of rotational speed had a greater impact on the modal response of the damaged engine rotor. In addition to the above research work, Heidari et al. [32], Dzenan [33], Cosme et al. [34], Sinha [35], and Jain [36] launched a series of modeling and simulation researches focused on blade off influence of components or whole engine, which could be referenced.

On the whole, there are many studies on the response of the blade and the whole engine to bird ingestion, but there are few reports on the dynamic response and safety analysis of the engine components during the period of the bird ingestion. On the basis of relevant research, this paper emphatically established the structure safety evaluation criteria of the engine bird ingestion, studied the dynamic response of the engine structure after bird ingestion, evaluated the safety of the whole structures after bird ingestion, and developed a structure safety analysis and assessment method of the engine bird ingestion.

\section{Criteria, Methods, and Procedures for Structural Safety Assessment of Bird Ingestion}

The effective 14 CFR Part 33.76 and $33.75 \mathrm{~g}(2)$ proposed the requirements of structural safety for large bird ingestion issues, which could be summarized to avoid the hazardous engine effects, as shown in Table 1.

According to the requirements for safety assessment of aeroengine bird ingestion in airworthiness regulations as well as the operating features and potential failure modes of aeroengines after large single-bird ingestion, the safety assessment objectives of rotor and load-carrying structures can be set as follows:

(1) Uncontained high-energy debris must not be generated

(2) Failure of the engine mount system leading to inadvertent engine separation must not happen

With the consideration of the effects of the above factors to the two assessment objectives, the fundamental causes 
TABle 1: Hazardous engine effects.

\begin{tabular}{ll}
\hline Effect & Remarks \\
\hline & (i) Uncontainment of high-energy debris \\
& (ii) Concentration of toxic products in the engine bleed air intended for the cabin sufficient to incapacitate crew or \\
passengers \\
(iii) Significant thrust in the opposite direction to that commanded by the pilot \\
Hazardous & (iv) Uncontrolled fire \\
& (v) Failure of the engine mount system leading to inadvertent engine separation \\
& (vi) Release of the propeller by the engine \\
(vii) Complete inability to shut the engine down
\end{tabular}

would be found out after in-depth analysis, as indicated in Figure 1 and Table 2.

Consequently, the safety assessment criteria for engine rotor and load-carrying structures under large single bird ingestion are listed as follows:

(1) Falling cuttings and fracture of rotor/stator should not happen; otherwise, the engine casing should be capable of containing high-energy debris subsequently

(2) Exposed parts of load-carrying structures should be capable of sustaining direct impact of the bird or bird carcass generated by fan cutting, and the peak stress should remain in the safety limit of parts

(3) Peak stress or loads of parts that are likely to be impacted by the bird of load-carrying structures should remain in the safety limit

In the above assessment criteria, the first one proposes the aim and requirements to deal with the traditional bird impact problem, and it is mainly aimed at the blade deformation, bending, falling cuttings, fracture, rub impact, and so on which are caused by the bird impact the blade, and there are two stages to apply the criterion. The strength of blade itself should be evaluated; firstly, if it satisfies the corresponding requirements after bird ingestion, then the second stage is considered. Otherwise, in order to guarantee the evaluation purpose, casing containment analysis should be launched if falling cuttings, fracture, or mass missing of stators due to the motion of blade shows up. The second criterion responses to the impact assessment of the fan downstream runner in the latest regulations of bird ingestion with the purpose of preventing hazardous events such as the bird hitting to the downstream of the plate, damaging the corresponding struts, pipelines, or control wiring, catching fire, and losing the thrust control. The third criterion is intended to avoid the structural failure and disconnection of installation joints and other hazardous events during the transformation of load after bird ingestion. In the compliance verification process of these criteria, corresponding compliance methods or combined methods should be adopted to achieve the purpose of verification. Calculation, modeling, and analysis are the most logical, cost-effective, and efficient approaches in the verification process. In conclusion, the analysis method combined with the calculation method (MC2) in engineering evaluation class can be applied to the safety analysis and evaluation of engine load-carrying structure under bird ingestion [37], as shown in Figure 2.

Bird ingestion is teased out firstly, followed by grasping the key research contents that must be carried out for the verification of safety assessment criteria, establishing compliance verification technology solutions in detail and putting forward the corresponding compliance methods or their combination. On this basis, finite element modeling, model checking, computing/analysis, and comprehensive compliance analysis work such as regularity and influencing factors analysis can be performed to obtain the dynamic response of the engine structure after bird ingestion. Furthermore, according to the safety assessment criteria of parts and components, judge the structure safety of parts or components, generate the test report, calculation report, and analysis report and so on; then submit these illustrative documents to administering authority. The structure safety of the whole engine after bird ingestion can be determined with the combination of bird ingestion compliance verification of the whole engine.

\section{Bird Ingestion Model of High Bypass Ratio Turbofan Engine}

In order to evaluate the structural safety of the whole engine during bird ingestion, the analysis work was carried out according to the above process. A finite element model of a typical turbofan engine with high bypass ratio was established refer to a model of engine blade off [38]. A single-stage wide-chord titanium alloy blade fan and a 0-21 fulcrum scheme were adopted. The inner ring of the front fulcrum was located in the middle of the fan shaft, and the outer ring was fixed with the inner side of the drum of the front fulcrum. The outer ring of the middle fulcrum was fixed on the inner side of the drum of the intermediate casing, and the inner ring was located on the outer side of the low pressure shaft. The outer ring of the rear fulcrum was located inside the drum of the supporting plate of the rear bearing frame, and the inner ring was located at the tail end of the low pressure shaft. The fan casing was connected with the intermediate casing through the main bearing frame support plate. The combustion chamber casing and the turbine casing were fixed at the rear section of the intermediate casing. The geometric and finite element models of the whole engine 


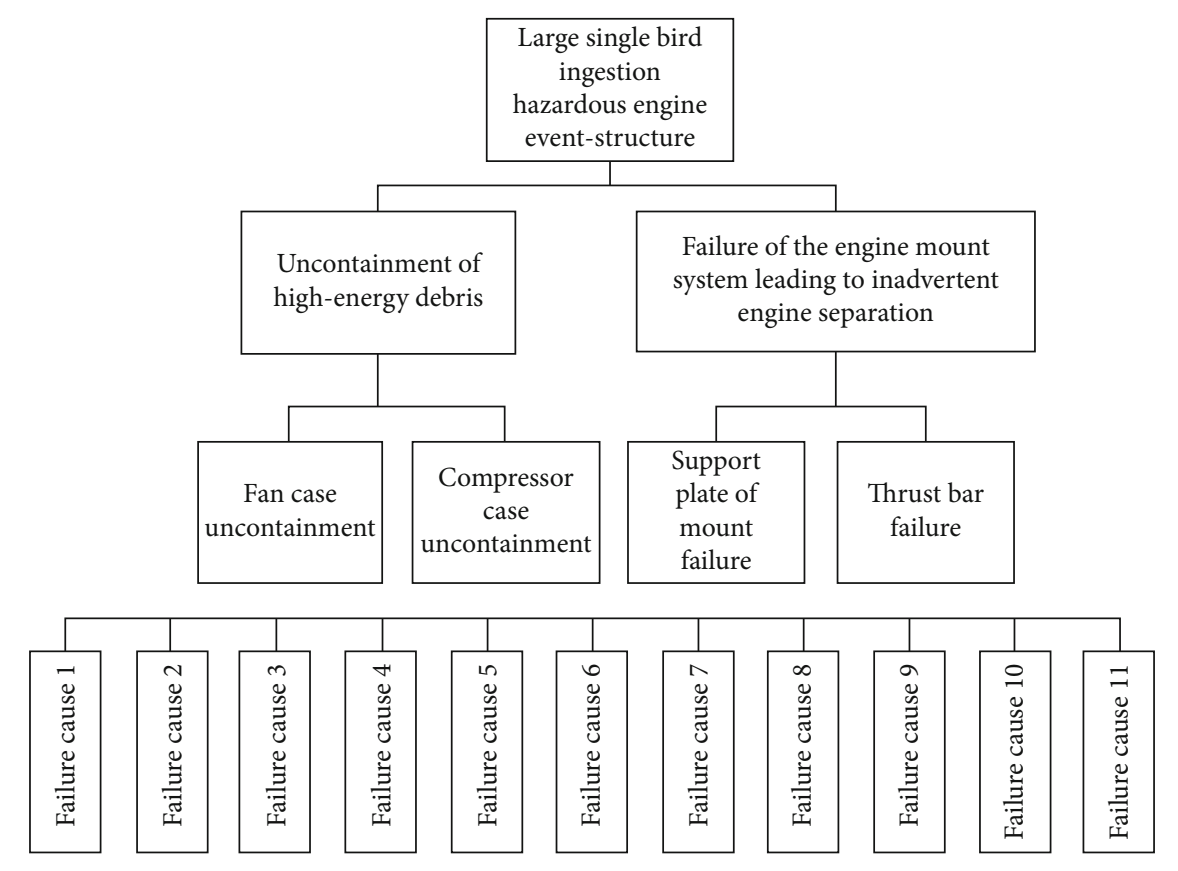

FIgURE 1: Fault tree analysis of bird ingestion.

TABLE 2: Reasons of failure.

\begin{tabular}{|c|c|}
\hline $\begin{array}{l}\text { Serial } \\
\text { number }\end{array}$ & Failure cause \\
\hline$\overline{1}$ & The birds impact the fan blade, causing it to fail and break off \\
\hline 2 & The birds impact the stationary part, falling cuttings and being thrown out due to the fan motion \\
\hline 3 & The birds impact the stationary/rotor part, falling cuttings and being thrown out \\
\hline 4 & The bird carcass impacts the blade, causing it to fail or fracture and be thrown out \\
\hline 5 & $\begin{array}{l}\text { Debris is generated due to the interaction of the upstream stationary/rotor part and bird ingestion, and the debris hit the } \\
\text { downstream rotor causing the debris to be thrown out }\end{array}$ \\
\hline 6 & The bird carcass impacts the primary support plate \\
\hline 7 & Debris of the upstream parts hits the primary support plate after bird ingestion \\
\hline 8 & The bird ingestion load transmits to the primary support plate directly \\
\hline 9 & The bird ingestion load transmits to the primary thrust bar \\
\hline 10 & The bird ingestion load transmits to the bearing block, causing it to fail \\
\hline 11 & Deformation of the drum and rotor binding shows up due to load transmission \\
\hline
\end{tabular}

including the intermediate casing, the main supporting plate, and the front and rear drums were established, as shown in Figures 3 and 4.

As shown in Figure 4, the whole engine grid model used 8-node hexahedron solid 164 element to divide entities and used 16 beam elements to simulate 4 hinges. The research team of the authors has carried out the grid sensitivity analysis to evaluate the response and damage of bird impact blades, in Yang's dissertation [39]; it was recommended that the element size reaches the level of $4-5 \mathrm{~mm}$, so that the deformation and damage analysis with high accuracy can be realized. In addition, in the similar research fields, Heidari et al. [32] used a simple method to divide a whole engine into shell elements and carried out the dynamic response analysis of the whole engine with the situation of blade off, the total number of elements was only 8256. In order to analyze the dynamic load of the whole engine under the condition of fan casing containment, Sinha [35] adopted a modeling scale of about 0.5 million elements and received good analysis results. Jain [36] established a fine finite element model of a rotor assembly with compressor/casing by using a scale of about 0.8 million elements and analyzed the transient load and damage better. The main purpose of this paper is to explore the load transmission and safety assessment of the whole engine at the moment of bird impact, and the research of its analysis method; therefore, after referring to the previous research work, in order to balance the accuracy and efficiency of calculation, the level of 5$10 \mathrm{~mm}$ of per element was selected to control the whole model scale to about 0.57 million elements, with 4970 


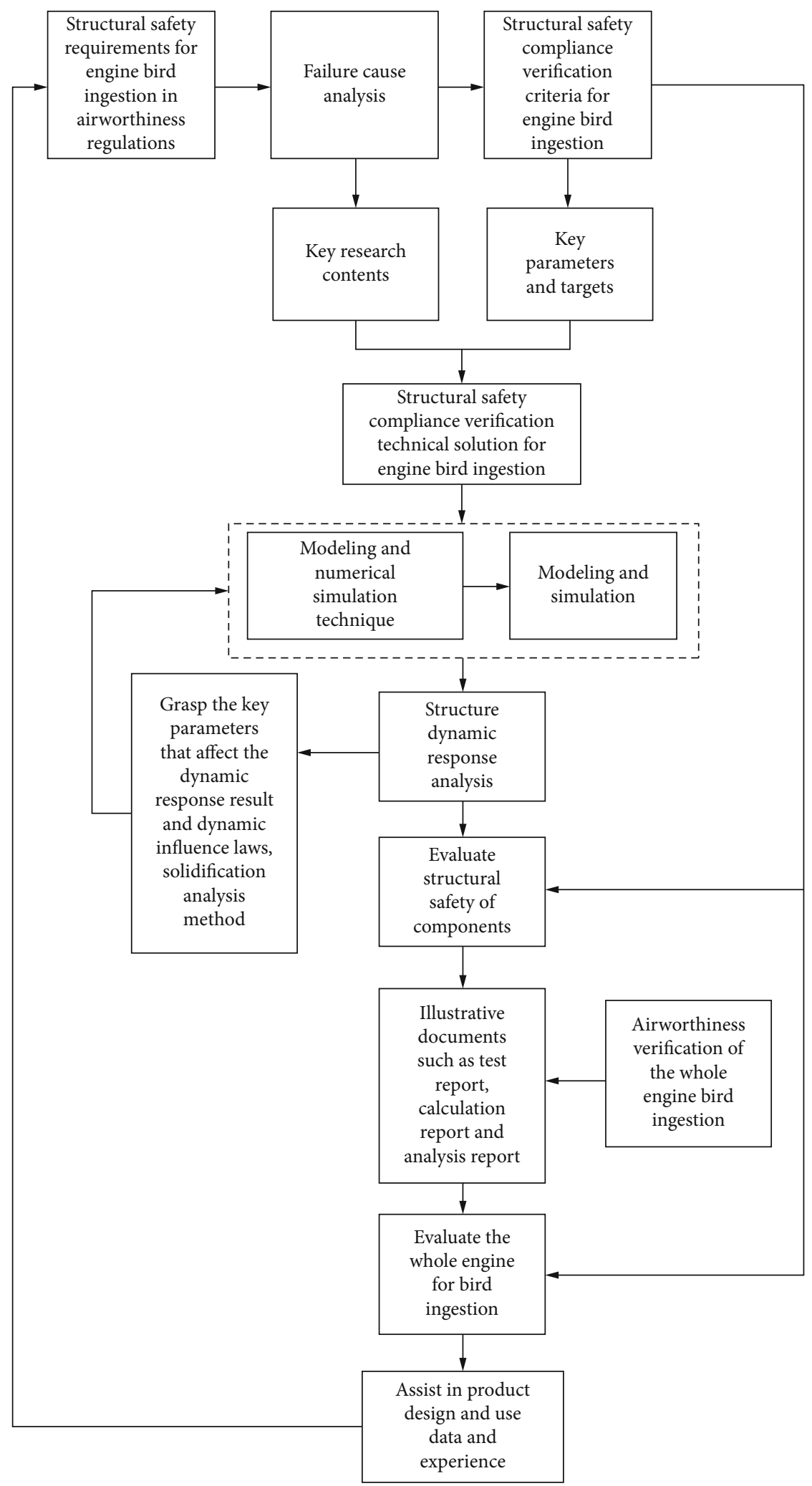

FIgURE 2: MC2 compliance verification method flow.

elements in each fan blade. It could not only represent the deformation and damage of the component to a certain extent but also described the dynamic response such as load and transmission.
The engine model referred to the material of real engine components, including TC4 titanium alloy, 2A70 aluminum alloy, and GH4169 super-alloy; the major parameters of Johnson-Cook material constitutive model 


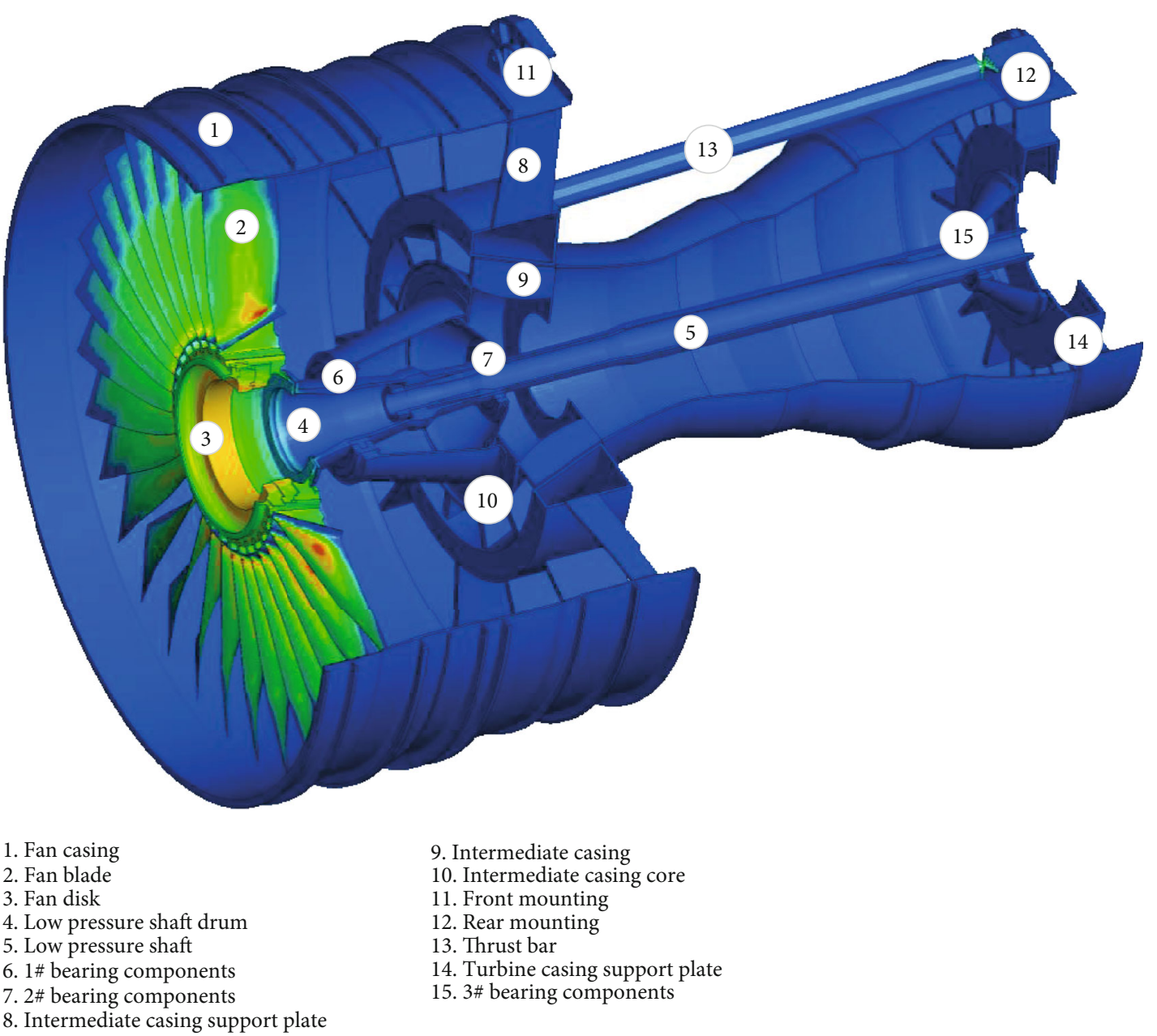

Figure 3: Geometric model profile of the whole engine.
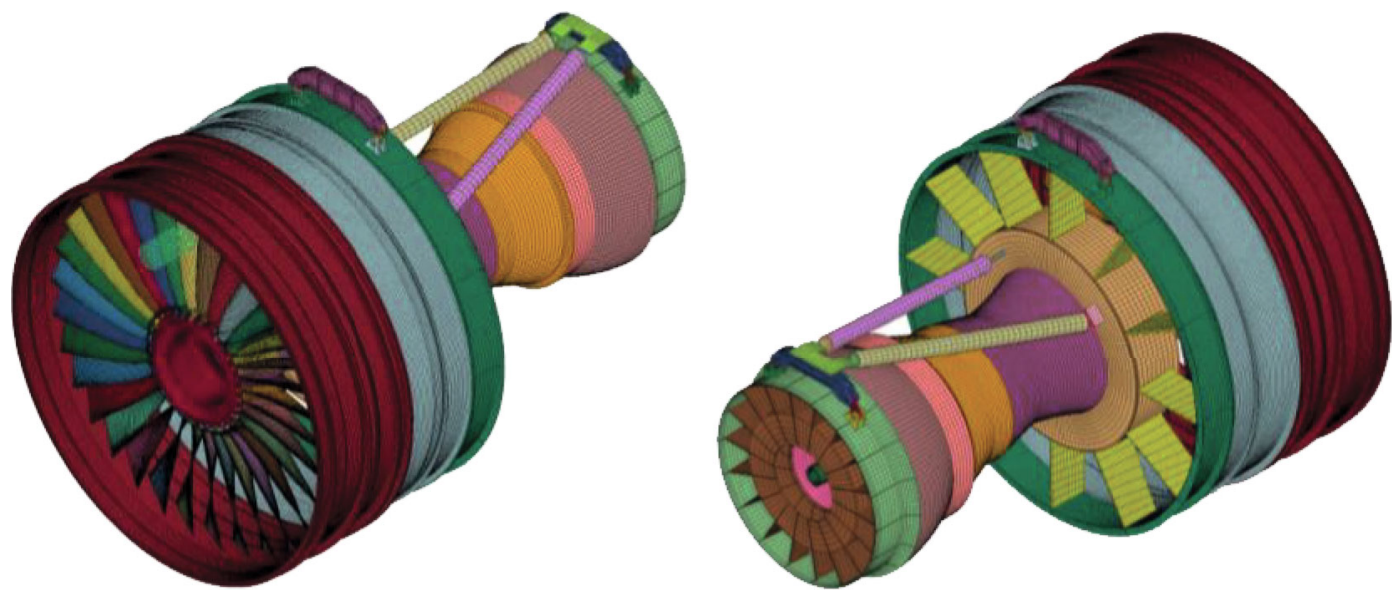

FIGURE 4: Finite model of whole engine.

are listed in Table 3 [38]. In calculation, referring to the hoisting mode of the real engine, the top surface of the front and rear mounting joints was fixed, and the freedom of all other components was released, and they were restrained by the contact surface. The node set of the fan blade was established to restrict the circumferential and radial displacement of the bottom node of the blade. The main body of the blade was not constrained, and the rotational speed of the blade was defined to rotate it. In order to simulate the running state of the engine rotor better after bird impact and maintain the power output of the rotor, the node set at the root position of the low- 
TABLE 3: Major parameters of Johnson-Cook material constitutive model.

\begin{tabular}{lccc}
\hline Parameter & TC4 titanium alloy & Value & GH4169 super-alloy \\
\hline Young's modulus & $E=113 \mathrm{GPa}$ & 2A70 aluminum alloy & $E=205 \mathrm{GPa}$ \\
Poisson's ratio & $\mu=0.33$ & $\mu=70 \mathrm{GPa}$ & $\mu=0.3$ \\
Density & $\rho=4430 \mathrm{~kg} / \mathrm{m}^{3}$ & $\rho=2800 \mathrm{~kg} / \mathrm{m}^{3}$ & $\rho=8240 \mathrm{~kg} / \mathrm{m}^{3}$ \\
Melting temperature & $T_{\mathrm{m}}=1878 \mathrm{~K}$ & $T_{\mathrm{m}}=923 \mathrm{~K}$ & $T_{\mathrm{m}}=1563 \mathrm{~K}$ \\
Heat capacity & $C_{\rho}=580 \mathrm{~J} / \mathrm{kg} * \mathrm{~K}$ & $C_{\rho}=795 \mathrm{~J} / \mathrm{kg} * \mathrm{~K}$ & $C_{\rho}=481 \mathrm{~J} / \mathrm{kg} * \mathrm{~K}$ \\
$A$ & $1089 \mathrm{MPa}$ & $364 \mathrm{MPa}$ & $1350 \mathrm{MPa}$ \\
$B$ & $1083 \mathrm{MPa}$ & $436 \mathrm{MPa}$ & $1139 \mathrm{MPa}$ \\
$C$ & 0.014 & 0.0054 & 0.0134 \\
$m$ & 1.1 & 1.398 & 1.03 \\
$n$ & 0.93 & 0.559 & 0.6522 \\
$D_{1}$ & -0.09 & 0.14 & 0.11 \\
$D_{2}$ & 0.27 & 0.136 & 0.35 \\
$D_{3}$ & 0.48 & -2.02 & 4.8 \\
$D_{4}$ & 0.014 & 0.0272 & 0 \\
$D_{5}$ & 3.87 & 0 & 0 \\
\hline
\end{tabular}

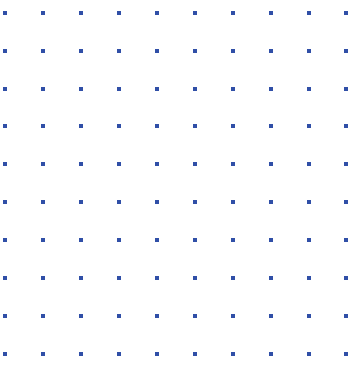

FIgURE 5: SPH model of a single $2.75 \mathrm{~kg}$ large bird.

TABle 4: Parameters of hydrodynamic elastic-plastic model.

\begin{tabular}{lc}
\hline Parameter & Value \\
\hline Density & $\rho=970 \mathrm{~kg} / \mathrm{m}^{3}$ \\
Shear modulus & $G=2.07 \mathrm{GPa}$ \\
Yield stress & $\sigma=0.02 \mathrm{MPa}$ \\
Plastic modulus & $\mathrm{Eh}=0.001 \mathrm{MPa}$ \\
\hline
\end{tabular}

pressure turbine on the shaft was set as the momentum output position, and the rotational speed of the whole rotor was defined as $4500 \mathrm{rpm}$ by the keyword $*$ Define curve.

In order to represent the initial state of the model, the keyword $*$ SPC_Set was used to define the fixed support of the installation section. The keyword $*$ Prescribed_montion_Set was defined to realize the low-pressure turbine power output. The keyword *Automatic_surface_to_Surface defined the contact of each rolling surface of the bearing sets. The connection surfaces of multiple fixed components were defined by the keyword *tied method. The keyword *eroding_nodes_to_ surface defined the bird's contact to the fan blade. The bulk viscosity and hourglass were controlled by default values. The calculation time was set to 13 milliseconds.

According to the size of the engine model and the requirements of the current effective airworthiness regulations, the mass of a single large bird ingested into the engine is $2.75 \mathrm{~kg}$, which is represented by a cylinder with an aspect ratio of 2 . The bird's diameter was $0.112 \mathrm{~m}$. The $\mathrm{SPH}$ method was used for modeling, as shown in Figure 5.

The bird model was based on hydrodynamic elasticplastic model and linear polynomial equation of state; the parameters of hydrodynamic elastic-plastic model [18] are listed in Table 4.

In the process of building the bird model, the linear polynomial equation of state (EOS) can be used to describe the large deformation characteristics of bird in combination with the hydrodynamic elastic-plastic model. EOS of bird is shown in Formula (1), compression ratio $\eta=\rho / \rho_{0}-1$, where $\rho$ and $\rho_{0}$ are the current density and initial density, respectively $\left(970 \mathrm{~kg} / \mathrm{m}^{3}\right)$.

$$
P=C_{0}+C_{1} \eta+C_{2} \eta^{2}+C_{3} \eta^{3}+\left(C_{4}+C_{5} \eta+C_{6} \eta^{2}\right) E
$$

It is very important to confirm the parameters related to the EOS of the bird. Therefore, a series of experiments and analytical studies have been carried out to simulate bird impact on rigid target with gelatin artificial bird [40, 41], as is shown in Figure 6.

According to the above research data, the pressure value of bird impact target plate center was measured and calculated, and the difference between the initial impact response and the constant flow response was taken as the objective function to carry out parameter optimization iteration, and 


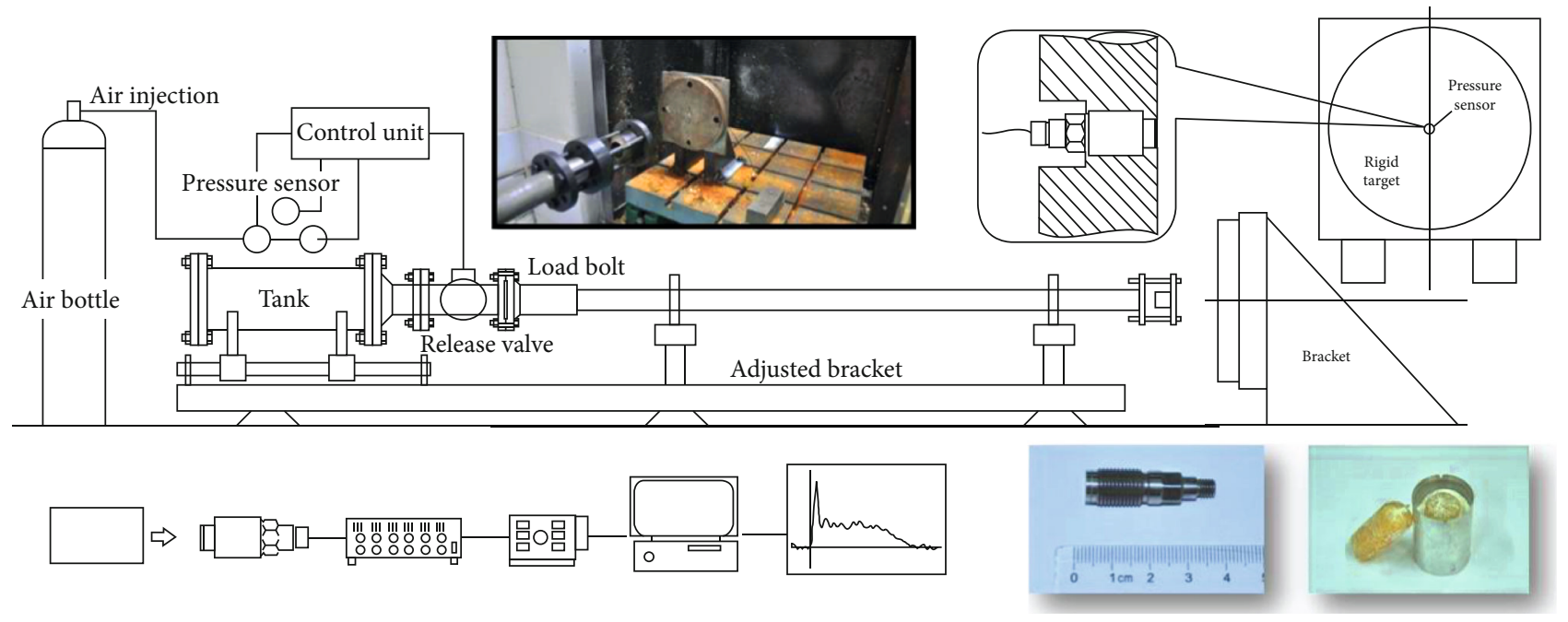

FIGURE 6: Experiments and analytical studies of gelatin artificial bird impact rigid target.

TABLE 5: Undetermined coefficient of the bird's EOS.

\begin{tabular}{lc}
\hline Parameter & Value \\
\hline$C_{0}$ & 0 \\
$C_{1}$ & $5 e 7$ \\
$C_{2}$ & $4.055 e 9$ \\
$C_{3}$ & $3.03 e 10$ \\
$C_{4}$ & 0 \\
$C_{5}$ & 0 \\
$C_{6}$ & -25 \\
\hline
\end{tabular}

the undetermined coefficient $\mathrm{c} 0-\mathrm{c} 6$ of the EOS was obtained, as listed in Table 5.

In order to ensure the rationality of the bird ingestion finite element model, the steady-state operation of the engine finite element model was checked before bird ingestion analysis was carried out. The centrifugal force was applied to the model fan by using the implicit calculation method provided by LS-DYNA, the keywords $*$ Implict_general and $*$ Implict_ solution were defined to realize the centrifugal force calculate, and the keyword *interface_springback_LS-dyna was defined to output DYNAIN binary files so as to build the centrifugal force field. The speed of $4500 \mathrm{rpm}$ was applied to the model fan to make the engine run in a steady state, as shown in Figure 7.

As shown in Figure 7, a single blade of the whole stage fan was selected and seven units are selected along the blade height direction. The stress on the element and the kinetic energy curve of a single blade were investigated. It could be seen that the engine model runs steadily. In order to solve several unstable problems in calculation, the following control methods and settings are adopted. It could effectively prevent the initial penetration of birds to blades by adjusting the contact stiffness factor from 0.1 to about 0.7. Due to the high-frequency response characteristics of bird impact, it was better to set the data output time interval above $1 e-5$ seconds. In the aspect of describing rotor rotation, the whole rotor entity speed was given by the keyword $*$ define_curve and rising rotating speed slowly to ensure the balance of internal load of rotor; however, the acceleration time of the rotor would increase dramatically and the calculation efficiency would decrease. In this simulation, the method of combining the definition of $*$ prescribed_montion_set and *define_curve was used to match the power output stable speed of the low-pressure turbine and the stable speed of the whole rotor. With the application of centrifugal prestress, the problem of long calculation time caused by slow application of rotating speed was avoided. The server used for calculation was configured as Intel Xeon dual core $\mathrm{CPU}$, with the main frequency of $2.1 \mathrm{GHz}$ and the computer memory of $32 \mathrm{~GB}$. It took about 27 hours and 40 minutes to complete the calculation of bird ingestion time of $13 \mathrm{~ms}$.

\section{Response and Structural Safety Analysis of Whole Engine during Bird Ingestion}

LS-Dyna was used to simulate the bird ingestion. The fan speed was set to $100 \%$ of the rotational speed. According to the airworthiness clause, the ingestion speed of a single bird is $103 \mathrm{~m} / \mathrm{s}$, and the impact position was estimated at 70\% of the blade height.

4.1. Fan Blade Bird Ingestion Process. Figure 8 shows the state of the bird being cut by the fan blade after it was ingested into the fan and the stress/strain status of 7 blades after bird impact.

As shown in Figure 8, the bird was cut by seven fan blades, and a great impact load was generated on the blades. See Figure 9 for the bird impact load generated on each blade.

Figure 10 shows the part of bird cut by each blade. See Table 6 for the slice mass after measurement and conversion. 

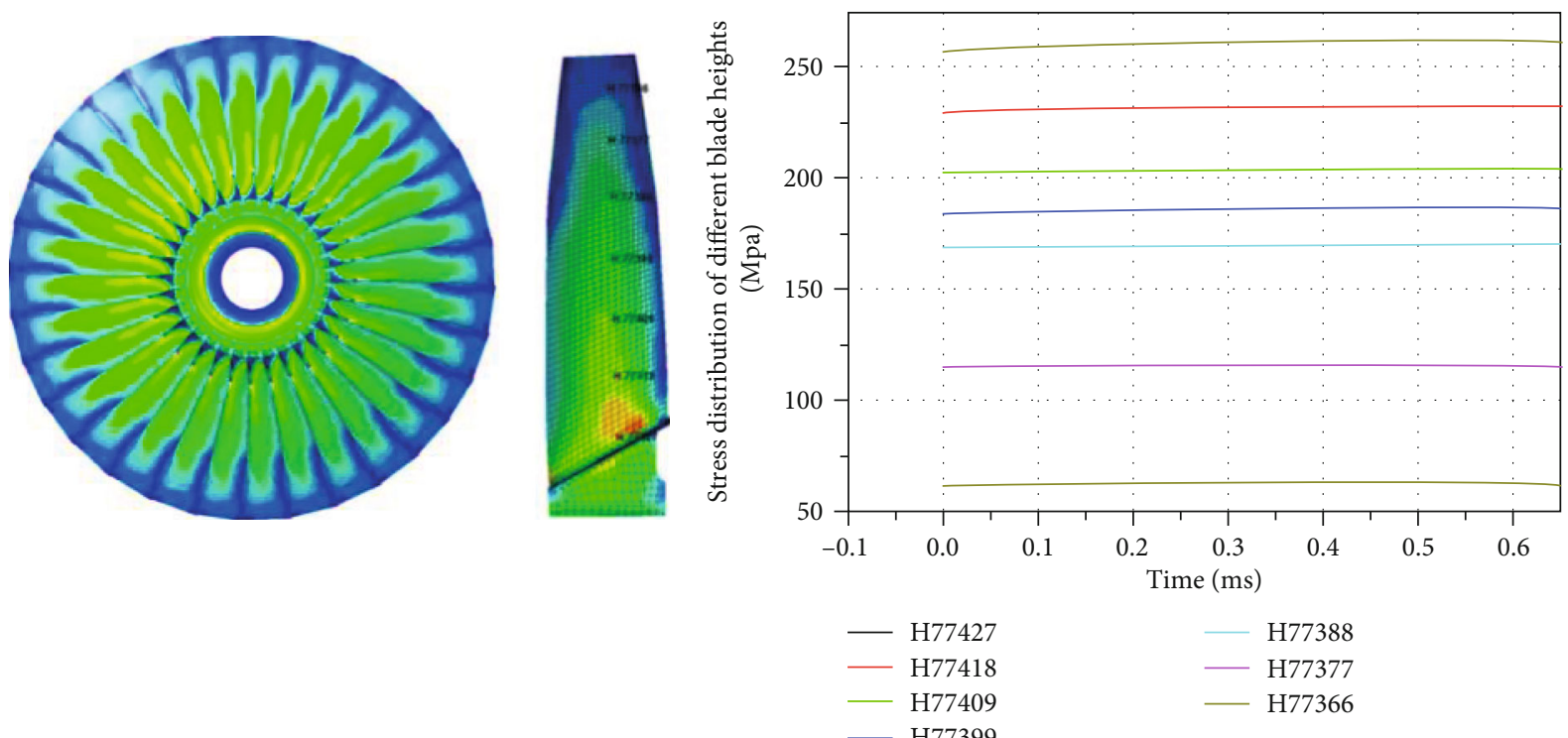

(a)

(b)

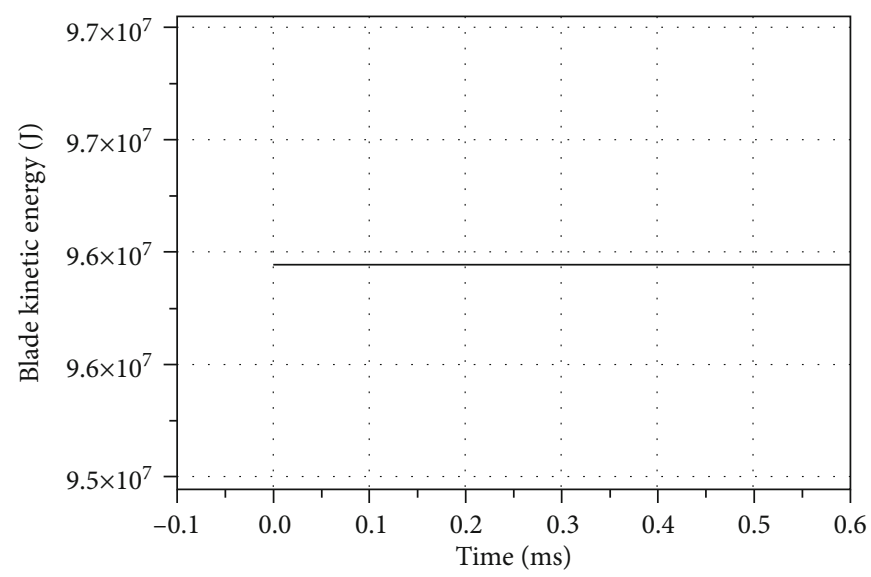

(c)

Figure 7: The state diagram of each component under steady-state operation: (a) stress distribution of blade, (b) stress of different blade heights, and (c) blade kinetic energy versus time.
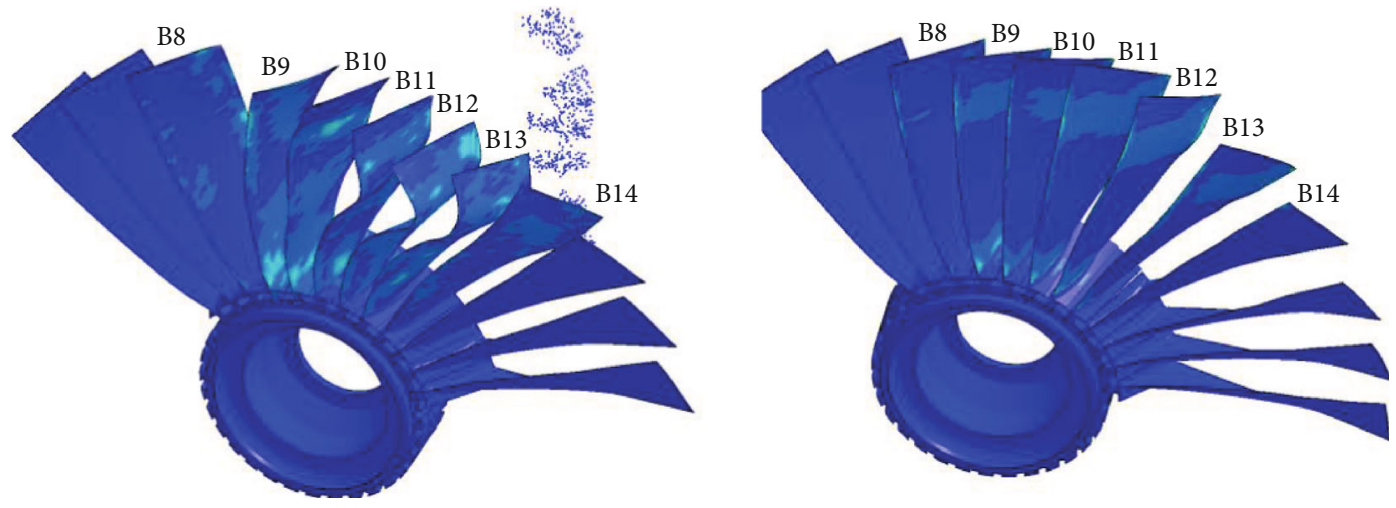

Figure 8: Bird slice and stress/strain status due to impact.

As shown in Figure 10 and Table 6, the load generated by bird impact on the blade was closely related to the slice mass. In general, with the slice mass of bird increasing, the impact load on the blade increased, and the degree of bending and plastic deformation on the blade increased as well. Among them, the mass of the seventh slice was 


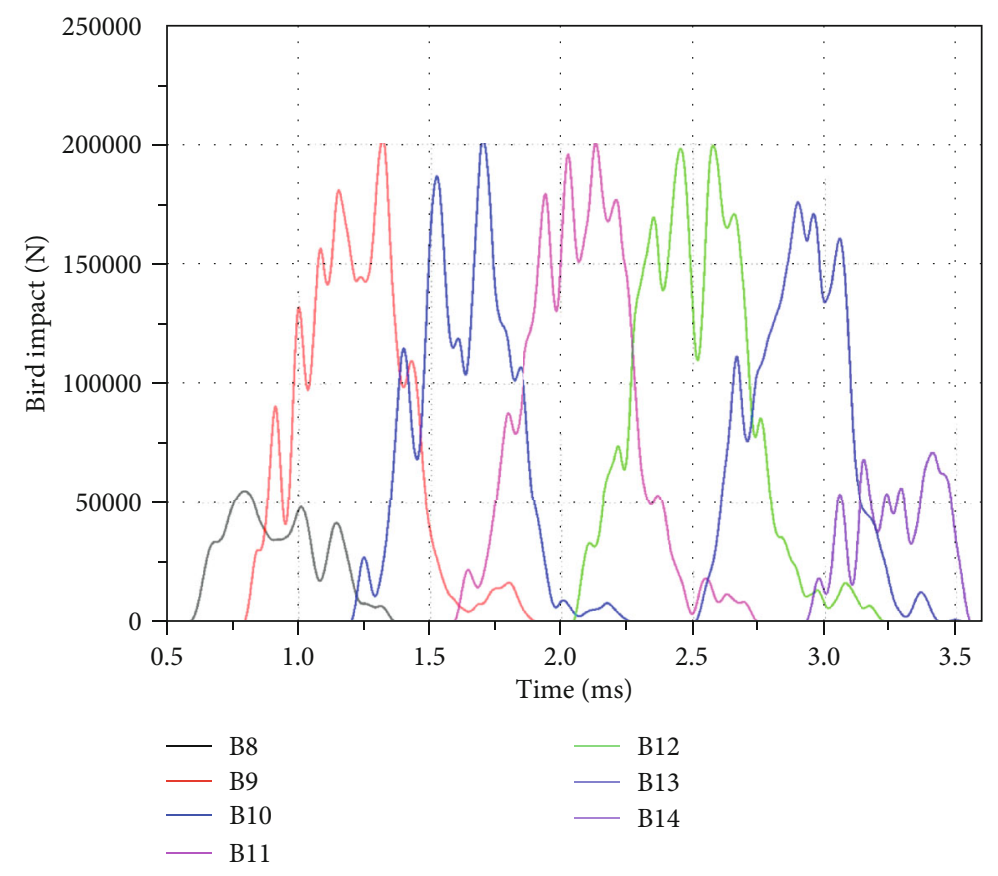

FIGURE 9: Bird impact force on relevant blades.

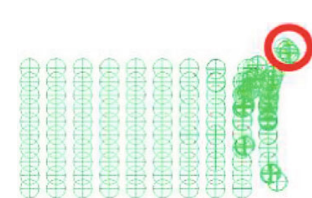

(a)

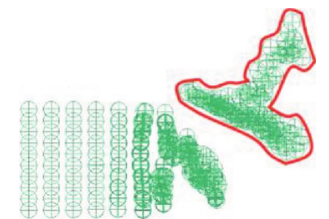

(b)

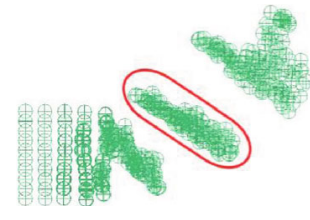

(c)

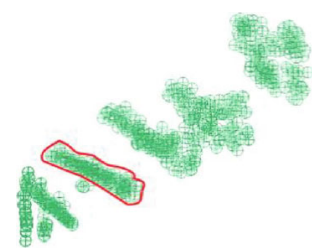

(e)

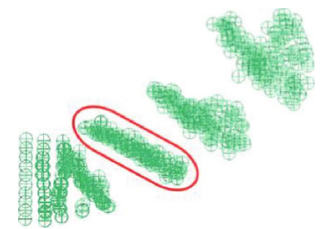

(d)

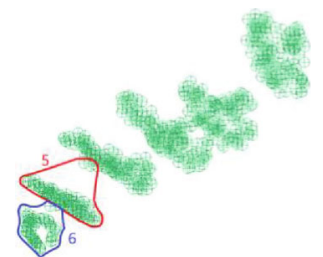

(f)

Figure 10: Bird impact fan blades slices: (a) Slice01, (b) Slice01+02, (c) Slice03, (d) Slice04, (e) Slice05, and (f) Slice06+07.

about 10 times that of the first slice, but the load state of the seventh blade was similar to that of the first blade, because the leading edge of the first blade was cut by the bird, while the leading edge of the seventh blade was not cut, and the collision effect between the bird slice and the blade was relatively slight.

Figure 11 shows the time-dependent curves of system internal energy and deformed blades' internal energy.
In this finite element analysis model, the kinetic energy of the rotor came from the preset momentum curve, so the energy was continuously flowing into the system, which was different from the simple kinetic energy-internal energy conversion. The change of the system internal energy was caused by the constant input kinetic energy and the energy disturbance and redistribution due to bird impact. The change mainly included the elastic-plastic deformation of 
TABLE 6: Mass of bird impact fan blade slices.

\begin{tabular}{lccccccc}
\hline Slices & 01 & 02 & 03 & 04 & 05 & 06 & 07 \\
\hline Particle number & 8 & 150 & 143 & 157 & 142 & 119 & 81 \\
Mass (g) & 27.5 & 515.63 & 491.56 & 539.69 & 488.12 & 409.06 & 278.44 \\
\hline
\end{tabular}

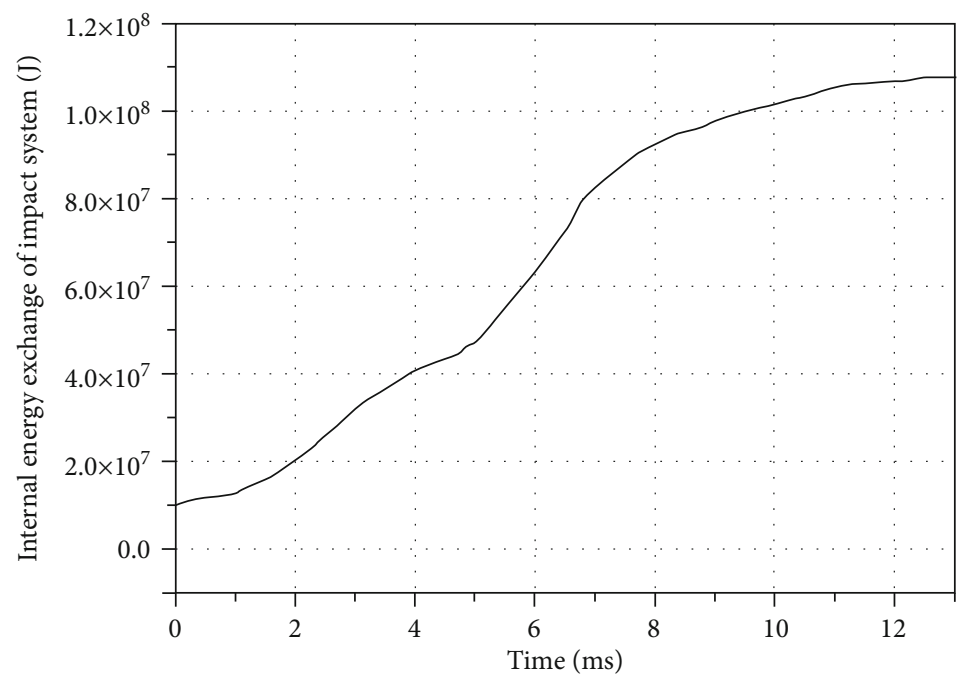

(a) Exchange of internal energy of system

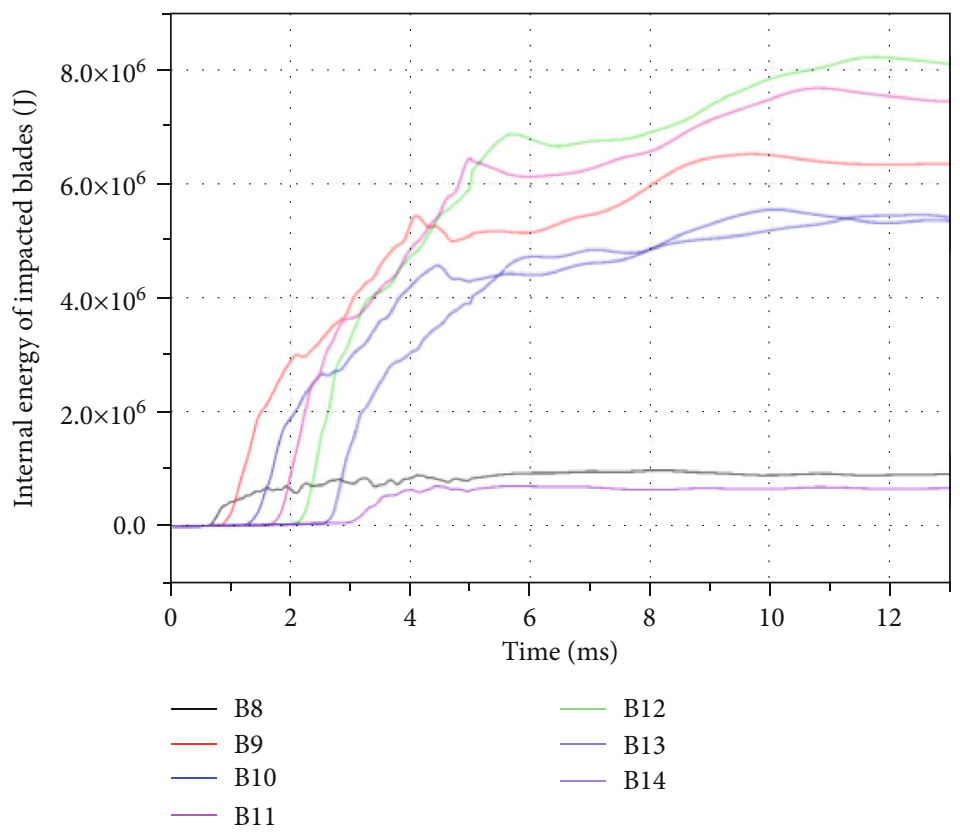

(b) Exchange of internal energy of deformed blades

FiguRE 11: Exchange of system internal energy and blades' internal energy: (a) exchange of internal energy of system and (b) exchange of internal energy of deformed blades.

the blades and the nonlinear losses of other components, such as vibration, deformation, and friction damping. The results showed that the elastic-plastic deformation of the blade absorbed about $35 \%$ of the internal energy increment of the system.
Figure 12 shows the dynamic load transfer mode and path due to bird impact.

As shown in Figure 12, when the bird impacted the fan blade, the impact load could be divided into the forward component $\mathrm{Fa}$ parallel to the axial direction and the 


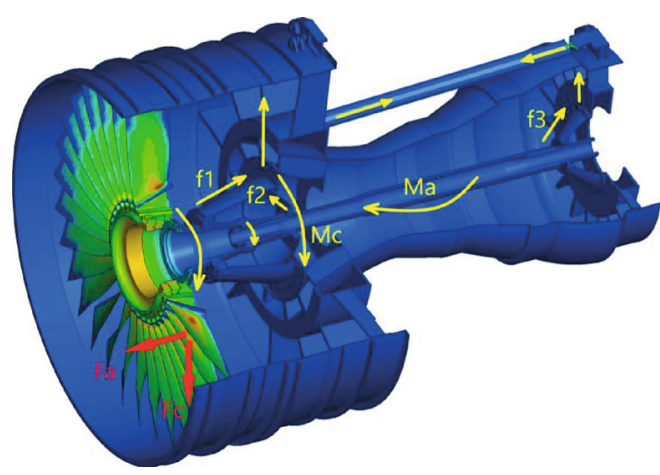

Figure 12: Dynamic load transfer mode and path due to bird impact.

circumferential component Fc opposite to the rotation direction, where Fa would cause the bending moment Ma of the rotor shaft and $\mathrm{Fc}$ would cause the torsional moment $\mathrm{Mc}$ of the connecting parts around the shaft system. Due to the combined effect of bending moment and torque, the dynamic response and dynamic load of the bearing pedestal would be produced. The dynamic loads $\mathrm{f} 1, \mathrm{f} 2$, and $\mathrm{f} 3$ were generated by transferring the load along the bracket and drum to the support plate and other components. Finally, after the interaction on the thrust bar, the load was transferred to the mounting of the engine. In the transmission process of bird impact load in the engine bearing frame, the shaft, drum, intermediate casing assembly, bearing assembly, thrust bar, and mounting were very important bearing parts.

4.2. Installation Joint-Thrust Bar Component Response. The curve of the maximum stress of the installation joint/thrust bar assembly with time is shown in Figure 13. It could be seen from the figure that after the fan ingested the bird, the load of bird impact was transmitted along the bearing frame, resulting in a sudden response on the front and rear mounting and thrust bar components. Among them, the response of the thrust bar was relatively light. The reason was that the thrust bar component was between the front and rear mounting, while the upper surface of the mountings was in a fixed state. Therefore, most of the impact energy transferred to this point was absorbed by the mountings, and only a small part of the impact energy forms a response fluctuation on the thrust bar; the response of the front mounting component was relatively larger and presented a higher frequency periodic vibration. The front mounting was close to the bird impact blade, and the load transfer frame composed of disk, shaft, drum, intermediate casing, support plate, etc. was more rigid, so the response amplitude and frequency were high; the rear mounting had the largest response and presents lowfrequency vibration, because the impact energy of bird impact caused a certain degree of rigid displacement of the whole engine, and the upper surface of the rear mounting was fixed, so the moment caused by the shaking of the whole machine acted on the rear mounting, resulting in a large and low frequency response inside the rear mounting. From the peak response in the figure, it can be seen that the impact energy was transmitted to the front mounting section at a slightly faster speed than to the rear mounting.

4.3. Medium Casing Component Response. As shown in Figure 14, when the bird started to impact the fan blade, it first caused the response of the core of the intermediate gearbox, which had a high response amplitude and showed a high frequency vibration trend in the rising process. This was due to the load fluctuation of the continuous cutting of the bird body by the blade during the bird impact. The impact energy of bird impact was directly transmitted to the outer ring of the bearing of the intermediate casing through the lowpressure shaft, so the response speed was the fastest and periodic; the response sequence of the support plate of the intermediate casing was basically the same as that of the intermediate casing, slightly slower than that of the core of the intermediate casing, and the bird started to impact the support plate of the intermediate casing after passing through the fan, resulting in an obvious impact peak (about $4.6 \mathrm{~ms}$ ) on the support plate. After the impact, the response level of the intermediate casing decreased gradually. The response amplitude of the intermediate casing was lower than that of the core and the support plate, because the intermediate casing was on the middle part of the bird impact load transfer path, and it did not directly take the bird impact, and there was a nonlinear connection surface between the intermediate casing and the drum, the support plate, the core, and other parts, Therefore, there was a certain energy dissipation in the process of energy transfer, so the response level was the lowest, and the main response was from the transfer of the support plate; the change rule was similar to that of the support plate. To sum up, the response mode of the intermediate casing assembly was the result of the combined action of the unbalanced force and bird impact force.

4.4. Response of Shafting Components. The curve of the maximum stress of shafting components with time is shown in Figure 15. During and after bird impact on the fan blade, the stress on the low-pressure shaft and drum of the lowpressure shaft presented obvious change. Among them, the stress level on the low-pressure shaft was higher than that on the drum of the low-pressure shaft, because in the early stage of bird impact, the impact energy transmitted from the blade to the disk was mainly absorbed by the lowpressure shaft drum, so the load amplitude of the drum changed greatly. However, because the drum was in the free cantilever state before the front fulcrum, the stress amplitude was less than the low-pressure shaft fixed by three fulcrum points. After the bird impact, the unbalanced force caused by the deformation of the fan blade further increased the response level of the low-pressure shaft in the three-point fixed state, while the stress level of the free end drum changes a little after the unloading of impact energy.

4.5. Contact Force of Bearing Assembly. Figure 16 shows the change trend of the contact force of three bearing components with time. As shown in the figure, the three groups of bearing contact force indicated obvious periodicity. Based 


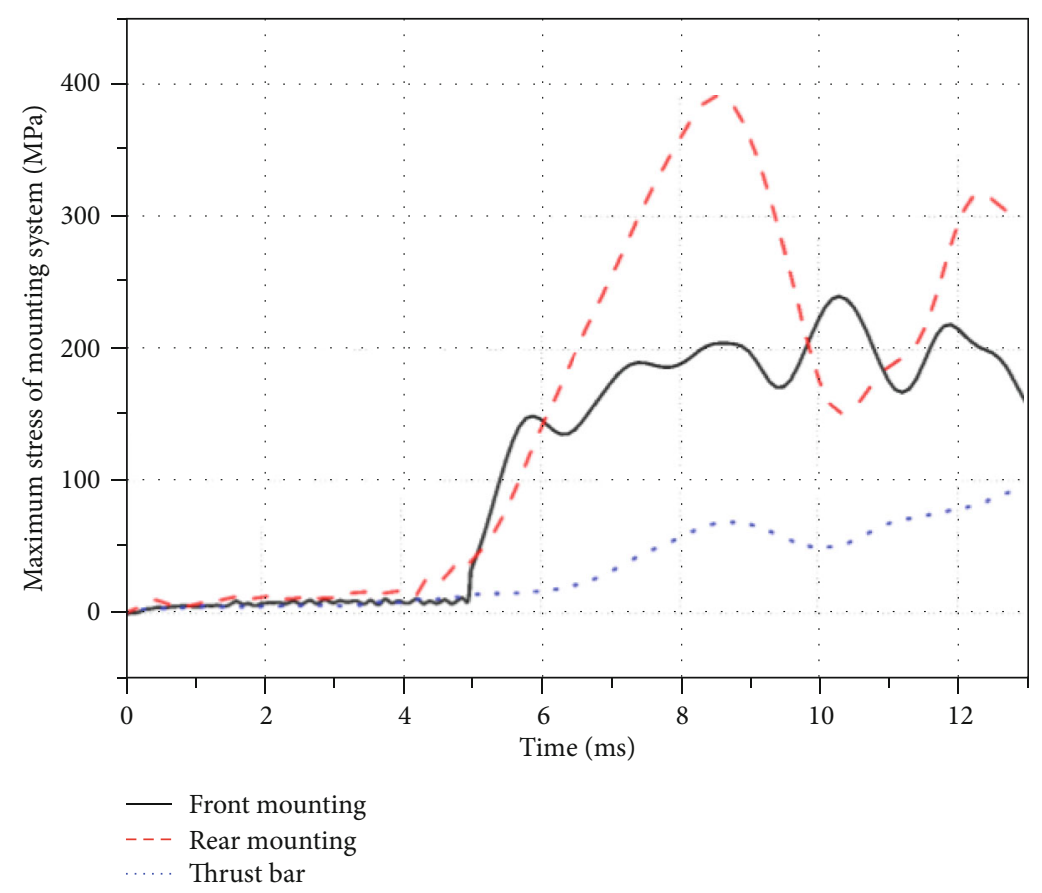

FIGURE 13: Stress variation of front mounting component.

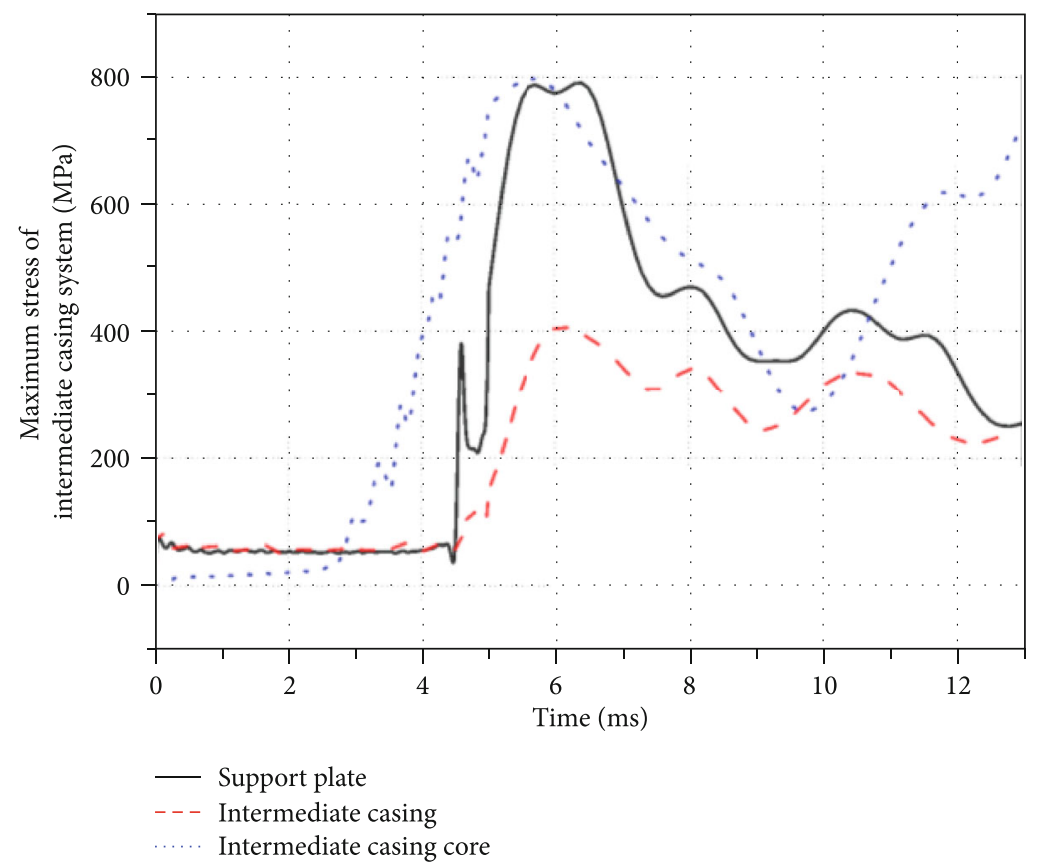

FIGURE 14: Stress variation of the support plate component of the intermediate casing.

on the change trend of $1 \#$ and $2 \#$ bearing contact forces, the period was about $13 \mathrm{~ms}$, which was consistent with the converted frequency of rotor speed $4500 \mathrm{rpm}$, indicating that the periodically changing contact force was due to the unbalanced load of the rotating rotor after bird impact. The variation amplitude of $1 \#$ bearing contact force was the largest; the main reason was that the bearing located closest to the fan blade; when the bird impacted the blade, the main impact load and unbalanced load form the first critical load trans- mission point, resulting in the highest response amplitude; the response time of $2 \#$ bearing was slightly later than $1 \#$ bearing's, and the response amplitude was also less than $1 \#$ bearing's; the reason was that $2 \#$ bearing was located further away from the fan disk than $1 \#$ bearing, the impact load and the unbalanced load on the fan disk needed more time to pass here, and the impact load and the unbalanced load have been unloaded by multiple components when passing through $1 \#$ bearing; 3\# bearing was the farthest away from the bird 


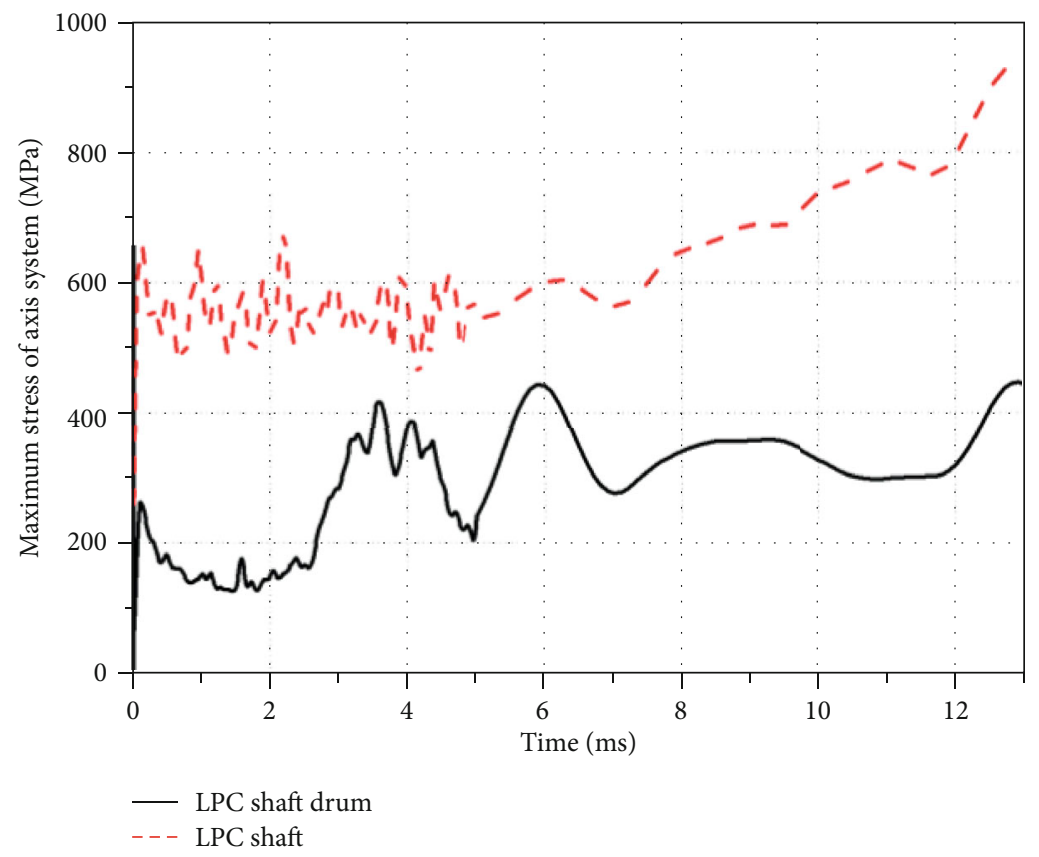

FIGURE 15: Stress variation of low pressure shaft drum component.

impact blade, so the response time moment was the latest, and most of the energy from the impact load and the unbalanced load has been absorbed after passing through almost the whole engine frame, so the amplitude of response and unbalanced load were relatively light. To sum up, the selection of $1 \#$ bearing was the most important issue during engine design.

4.6. Safety Assessment. After the numerical simulation of engine bird ingestion, the response of the key components in the model is as shown in Table 7.

As could be seen from the table above, the maximum stress level in the shock response of all components did not exceed the yield stress level of the selected material and was assessed according to the following criteria:

(1) No shedding or breaking of rotor/stator parts shall occur. In case of breakage, the casing shall be capable of containing the above high-energy debris. From the results of calculation and analysis, it was shown that there was plastic deformation of the fan blade due to bird impact, the plastic deformation of fan blade did not lead to the failure case of fan blade such as blade droppings and fractures, and there was no interaction between blades and blades and between blades and stationary parts. There was no failure of the stationary parts such as blade dropping, which met the basic requirements, so no need to carry out the analysis and verification of the casing containment capability

(2) All the exposed components in the load-carrying structures shall be able to withstand the direct impact of the corresponding bird carcass or the fragments of the bird body cut by the fan, and the peak stress level should be kept within the safety limit of the component. The results of calculation and analysis showed that none of the seven fan blades impinged by the birds has falling cuttings or fracture failure, and the outlet strut of the fan casing was also impacted by the bird fragments; although the stress level was relatively high, it has reached $770 \mathrm{MPa}$, but it has not exceeded the yield limit of the corresponding material, and failure cases have not occurred such as block dropping and fracture. Therefore, it could be judged that the engine structure has passed the safety assessment of the second evaluation criterion by calculation and analysis

(3) The peak stress or load level of all the components in the load-carrying structures which may be subjected to the bird impact load transmission should be kept within the safety limit of the parts while the engine sucked the bird

From the results of calculation and analysis, most components of the engine carrying structure have dynamic response due to the transfer of shock load during the bird ingestion, and the stress level of most components has majorly increased or fluctuated. However, the maximum stress level of each key component did not exceed the strength limit of the corresponding component material, which indicated that the instantaneous structural safety of each component could be ensured during the bird ingestion. The only potentially hazardous location is the $1 \#$ bearing assembly, which needs to be evaluated for the radial bearing capacity selected in the design. 


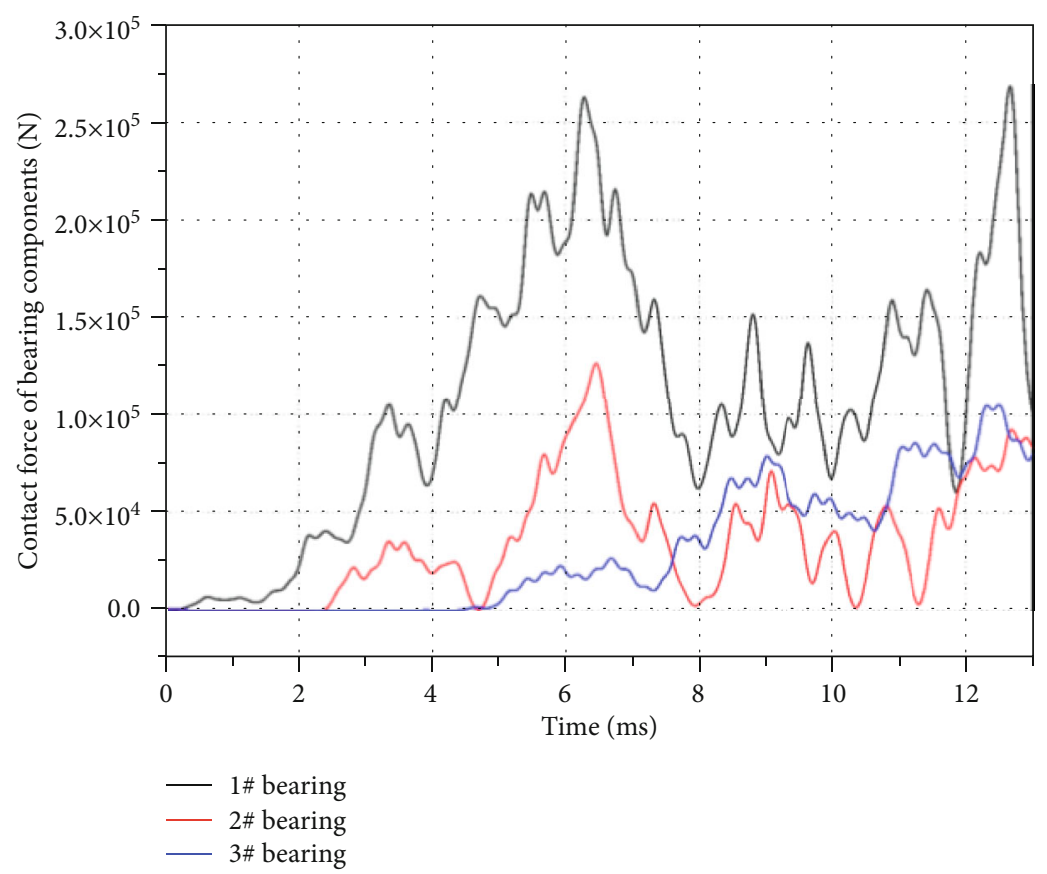

FIGURE 16: Contact force variation of bearing components.

TABLE 7: Response of key components in engine bird ingestion.

\begin{tabular}{lc}
\hline Component name & Maximum stress or load \\
\hline Front mounting joint & $240 \mathrm{MPa}$ \\
Rear mounting joint & $391.5 \mathrm{MPa}$ \\
Thrust bar & $96.3 \mathrm{MPa}$ \\
Intermediate casing support & $791 \mathrm{MPa}$ \\
Intermediate casing & $406 \mathrm{MPa}$ \\
Intermediate casing core & $797.3 \mathrm{MPa}$ \\
Low pressure shaft drum & $444 \mathrm{MPa}$ \\
Low pressure shaft & $940 \mathrm{MPa}$ \\
Front fulcrum bearing assembly & $2.68 e 5 \mathrm{~N}$ \\
Intermediate bearing assembly & $1.26 e 5 \mathrm{~N}$ \\
Rear fulcrum bearing assembly & $1.05 e 5 \mathrm{~N}$ \\
\hline
\end{tabular}

According to the above analysis, the simulation results of the bird ingestion model of high bypass ratio turbofan engine ingested a single large bird which showed that the engine was structurally safe during bird ingestion. The results of its analysis/calculation could be submitted as key technical documents to the airworthiness authorities for validation or to be references.

\section{Conclusion}

This paper established the criterion, method, and flow chart of structural safety evaluation for engine bird ingestion. The numerical simulation was carried out for the whole engine model ingesting a single big bird. The response changes of key bearing components in the model during bird ingestion are studied and analyzed. The struc- tural safety of engine bird ingestion was evaluated. The main conclusions are as follows:

(1) Based on the analysis of bird ingestion failure of engine, the safety assessment criteria for bird ingestion structure of engine were established as follows: (1) no block or breakage of rotor/stator components should occur; if it occurs, the casing should be able to contain the above-mentioned high-energy debris; (2) all exposed components in the load-carrying structure should be able to withstand the direct impact of bird body or bird body debris cut by fan, and the peak stress level should be equal to the corresponding bird body or bird body debris cut by fan. Maintain the safety limit of the components. (3) The peak stress or load level of all components in the load-carrying structure that may be subjected to bird impact load transmission should be kept within the safety limit of the components when the engine sucks birds

(2) A method and process of safety analysis and assessment for bird ingestion structure of turbofan engine based on analysis/calculation were proposed. The process included fault cause analysis, technical scheme determination, modeling and numerical simulation, dynamic response analysis, and structural safety assessment

(3) The finite element model of turbofan engine with large bypass ratio could reasonably describe the mechanical characteristics of engine steady-state operation. The bird ingestion finite element model of the whole engine could well simulate the process and dynamic response of the engine ingesting a single 
big bird. Through the analysis of the response process and response level of the key components, the structural safety of the engine ingesting a bird was evaluated according to the criteria. The results could be submitted to the airworthiness authorities for approval as an important reference

(4) Analytical and computational studies showed that the safety analysis and evaluation method of bird ingestion structure of turbofan engine based on analysis/calculation could be used to analyze and predict the airworthiness of bird ingestion structure of turbofan engine

\section{Data Availability}

The test data used to support the findings of this study are included within the article.

\section{Conflicts of Interest}

The authors declare that they have no conflicts of interest.

\section{Acknowledgments}

This work was supported by the National Science and Technology Major Project (2017-IV-0006-0043).

\section{References}

[1] A. S. Boyd, R. T. Murphy, and C. Gurney, "Aircraft accident Report on the Accident to Easten Air Lines, Inc, Lockheed Electra L-188, N5533 at Logan International Airport, Boston, Massachusetts et al.," Aircraft Accident Report Civil Aeronautics Board, 1960.

[2] National Transportation Safety Board, "Aircraft accident Report on the Accident to Overseas National Airways, Inc, Douglas DC-10-30, N1032F at JFK International Airport, Jamaica, New York," NTSB-AAR-76-19, 1975.

[3] National Transportation Safety Board, "Aircraft Accident Report: Loss of Thrust in Both Engines After Encountering a Flock of Birds and Subsequent Ditching on the Hudson River, US Airways Flight 1549, Airbus A320-214, N106US, Weehawken, New Jersey," NTSB-AAR-10-03, 2010.

[4] "Boeing 747-136 Bird Ingestion Accident," Aircraft Accident Report, Air Accidents Investigation Branch, London (Heathrow) Airport, 1997.

[5] "Boeing 767-300 Bird Ingestion Accident," Aircraft Accident Report, National Transportation Safety Board , NTSBATL07CA051, Chicago Airport, 2007.

[6] European Union Aviation Safety Agency, Certification Specification for Engine CS-E, European Union Aviation Safety Agency, 2009.

[7] US Federal Aviation Administration, FAA AC33.76, Airworthiness Standards: Aircraft Engines, Subpart E-Design and Construction; Turbine Aircraft Engines, Bird ingestion, Federal Aviation Administration, 2009.

[8] Advisory Circular, 33 76-1A, Bird Ingestion Certification Standards, Federal Aviation Administration, 2009.
[9] L. Castelletti and M. Anghileri, "Bird strike. The influence of the bird modeling on the slicing forces," in Proceedings of 31st European Rotorcraft Forum, Florence, Italy, 2005.

[10] J. Frischbier and A. Kraus, Multiple stage turbofan bird ingestion analysis with ALE and SPH methods, AIAA Paper, 2005.

[11] G. Yupu, Z. Zhenhua, C. Wei, and G. Deping, "Foreign Object Damage to Fan Rotor Blades of Aeroengine Part I: Experimental Study of Bird Impact," Chinese Journal of Aeronautics, vol. 20, no. 5, pp. 408-414, 2007.

[12] R. H. Mao, S. A. Meguid, and T. Y. Ng, "Finite element modeling of a bird striking an engine fan Blade," Journal of Aircraft, vol. 44, no. 2, pp. 583-596, 2007.

[13] S. A. Meguid, R. H. Mao, and T. Y. Ng, "FE analysis of geometry effects of an artificial bird striking an aeroengine fan blade," International Journal of Impact Engineering, vol. 35, no. 6, pp. 487-498, 2008.

[14] R. H. Mao, S. A. Meguid, and T. Y. Ng, "Effects of incidence angle in bird strike on integrity of aero-engine fan blade," International Journal of Crashworthiness., vol. 14, no. 4, pp. 295-308, 2009.

[15] Y. N. Shmotin, P. V. Chupin, D. V. Gabov et al., "Bird strike analysis of aircraft engine fan," in 7th European LS-DYNA users conference, Salzburg Austria, 2009.

[16] M. Selezneva, P. Stone, T. Moffat, K. Behdinan, and C. Poon, "Modeling bird impact on a rotating fan: the influence of bird parameters," Aerospace Journal, vol. 4, 2012.

[17] R. Prakash, H. Channegowda, and A. Kaliyaperumal, A study on bird impact damages on shrouded fan blades of an aeroengine ASME 2013 Gas Turbine India Conference, American Society of Mechanical Engineers, 2013, V001T05A022V001T05A022.

[18] R. Vignjevic, M. Orłowski, T. De Vuyst, and J. C. Campbell, “A parametric study of bird strike on engine blades," International Journal of Impact Engineering, vol. 60, pp. 44-57, 2013.

[19] S. A. Howard, J. T. Hammer, K. S. Carney, and J. M. Pereira, "Jet Engine Bird Ingestion Simulations: Comparison of Rotating to Non-Rotating Fan Blades," NASA/TM-2013-217904, National Aeronautics and Space Administration, Cleveland, OH, USA, 2013.

[20] A. Siddens and J. Bayandor, "Multidisciplinary impact damage prognosis methodology for hybrid structural propulsion systems," Computers and Structures, vol. 122, pp. 178-191, 2013.

[21] A. J. Siddens and J. Bayandor, "Detailed post-soft impact progressive damage analysis for a hybrid structure jet engine," in 28th International Congress of the Aeronautical Sciences, vol. 33no. 2, pp. 122-145, Brisbane, Australia, 2014.

[22] L. Jun and L. Yulong, "Numerical simulation of a rotary engine primary compressor impacted by bird[J]," Chinese Journal of Aeronautics, vol. 26, no. 4, pp. 926-934, 2013.

[23] K. M. Handschuh, S. G. Miller, M. J. Sinnott et al., "Materials, manufacturing and test development of a composite fan blade leading edge subcomponent for improved impact resistance," Japanese Journal of Acute Medicine, vol. 34, no. 2, pp. 485489, 2014.

[24] S. G. Miller, K. M. Handschuh, and M. J. Sinnott, "Materials, manufacturing, and test development of a composite fan blade leading edge sub-component for improved impact resistance," NASA/TM-2015-218340, NASA Glenn Research Center, Cleveland, OH, USA, 2015. 
[25] Z. Chuana, J. Xiang-hua, C. Xiang-hai, and S. Tong-cheng, "TC4 Hollow Fan Blade Structural Optimization Based on Bird-Strike Analysis," Procedia Engineering, vol. 99, no. 2015, pp. 1385-1394, 2014.

[26] D. Zhang and Q. Fei, "Effect of bird geometry and impact orientation in bird striking on a rotary jet-engine fan analysis using SPH method," Aerospace Science and Technology, vol. 54, pp. 320-329, 2016.

[27] S. K. Sinha, "Transient dynamics of slicing-impact loading on jet engine fan blades during a bird-strike event," in 15th International LS-DYNA Users Conference, Detroit, June 2018Paper No. 3.

[28] Ramachandra, "Evaluation of transient engine-bearing-loads due to bridstrikes," in Gas turbine research establishment ministry of defence, pp. 42-45, Government of India, 1996.

[29] W. Czeslaw, A. Samy, T. Vijay, and B. Arjaan, "Fan blade optimization under medium bird strike load," in Virtual product development conference, Huntington Beach,California, 2004.

[30] Liebich, "A full size roter dynamic test rig for whole engine mechanics of areoengines," in Chair of engineering design and product reliability, vol. 8, no. 3pp. 137-144, Berlin Institute of Techology, Germany, 2012.

[31] S. Badshah, A. Naeem, A. F. Rafique, I. U. Haq, and S. A. Malik, "Numerical study on the critical frequency response of jet engine rotors for blade-off conditions against bird strike," Applied Sciences, vol. 9, no. 24, p. 5568, 2019.

[32] M. Heidari, D. Carlson, S. Sinha et al., "An efficient multidisciplinary simulation of engine fan-blade out event using MD nastran," in Proc. 49th AIAA/ASME/ASCE/AHS/ASC Structures, Structural Dynamics and Materials Conf, pp. 187189, Schaumburg, IL, 2008.

[33] H. Dzenan, Mechanical loads on a turbofan engine structure at blade-off, Lulea University of Technology, 2009.

[34] N. Cosme, D. Chevrolet, J. Bonini, B. Peseux, and P. Cartraud, "Prediction of engine loads and damages due to fan blade off event," in Structural Dynamics, and Materials Conference, pp. 190-194, Denver, USA, 2002.

[35] S. K. Sinha and S. Dorbala, "Dynamic loads in the fan containment structure of a turbofan engine," Journal of Aerospace Engineering, vol. 22, no. 3, pp. 260-269, 2009.

[36] R. Jain, "Prediction of transient loads and perforation of engine casing during blade-off event of fan rotor assembly," in Proceedings of the IMPLAST 2010 Conference, Rhode Island USA, 2010.

[37] G. Luo, Structural safety analysis and assessment method of high bypass ratio turbo-fan engine due to bird ingestion, Nanjing University of Aeronautics and Astronautics, 2017.

[38] J. Wu, "Analysis of whole engine response due to blade out load of aero-engine," Nanjing University of Aeronautics and Astronautics, 2016.

[39] J. Yang, Research on response of fan blade subjected to bird strike and aerodynamic performance of damaged fan, Nanjing University of Aeronautics and Astronautics, 2014.

[40] S. Bin, Study on identification of artificial bird's material parameter based on rigid target impact test, Nanjing University of Aeronautics and Astronautics, 2014.

[41] G. Luo, C. Wei, L. Lulu, and Z. Zhenhua, "Experimental investigation of small artificial bird impact on rigid target," in The 2nd Aerospace Structural Impact Dynamics International Conference, Sevilla, Spain., November 2015. 